\title{
Making economic sense of brain models: a survey and interpretation of the literature
}

\section{Werner Neu}

On p. 177, the superscript to footnote 15 should be moved to line 4 after the words "philosophy of mind".

On p. 187, Chap. 4.2 line 14, the words "at the basis of institutions" should read "at the base of institutions".

In Reference Kosfeld et al. the word "Narue" should read "Nature".

The online version of the original article can be found under doi:10.1007/s10818-008-9038-5. 